\begin{tabular}{|c|l|}
\hline Title & Quantized conductance behavior of Pt metal nanoconstrictions under electrochemical potential control \\
\hline Author(s) & Konishi, Tatsuya; Kiguchi, Manabu; Murakoshi, Kei \\
\hline Citation & $\begin{array}{l}\text { Surface Science, 601(18), 4122-4126 } \\
\text { https://doi.org/10.1016/.Susc.2007.04.071 }\end{array}$ \\
\hline Issue Date & 2007-09-15 \\
\hline Doc URL & http://hdl.handle.net/2115/29766 \\
\hline Type & article (author version) \\
\hline File Information & SS601-18.pdf \\
\hline
\end{tabular}

Instructions for use 


\section{Quantized conductance behavior of Pt metal nano constrictions under electrochemical potential control}

Tatsuya Konishi, Manabu Kiguchi, and Kei Murakoshi ${ }^{1}$

Division of Chemistry, Graduate School of Science, Hokkaido University, Sapporo, 060-0810, Japan

We studied the quantized conductance behavior of mechanically fabricated Pt nanoconstrictions under electrochemical potential control in $\mathrm{H}_{2} \mathrm{SO}_{4}, \mathrm{Na}_{2} \mathrm{SO}_{4}$ and $\mathrm{NaOH}$ solutions. There was no clear feature in the conductance histogram, when the electrochemical potential of the nanoconstrictions was kept at the double layer or the under potential deposited hydrogen potential. At the hydrogen evolution potential, the conductance histograms showed clear features around 0.5 and $1 G_{0}$ in the $\mathrm{H}_{2} \mathrm{SO}_{4}$ solution. In $\mathrm{Na}_{2} \mathrm{SO}_{4}$ and $\mathrm{NaOH}$ solutions, a $1 G_{0}$ feature with a shoulder appeared in the histogram. The quantized conductance behavior of Pt nanoconstrictions could be controlled by the electrochemical potential and solution $\mathrm{pH}$.

Keywords: Scanning tunneling microscopy, Electrochemical potential, Conductance quantization, Point contact, Hydrogen, Platinum

\footnotetext{
${ }^{1}$ Correspondence author: Kei Murakoshi; Address: Division of Chemistry, Graduate School of Science, Hokkaido University, Kita 10, Nishi 8, Sapporo, Hokkaido, 060-0810, Japan; TEL+81 11706 2704; Fax: +81 11706 4810; E-mail: kei@sci.hokudai.ac.jp
} 


\section{Introduction}

In recent years, metal nanoconstrictions have attracted attention for interest of fundamental science as well as expectation of applications for nano devices $[1,2]$. The electrical conductance through an atomic scale metal nanoconstriction is expressed by $G=\left(\mathbf{2} \boldsymbol{e}^{2} / \boldsymbol{h}\right) \Sigma T_{i}$, where $T_{i}$ is the transmission probability of the $i$-th conductance channel, $e$ is the electron charge, $h$ is Planck's constant, and $G_{0}=2 e^{2} / h$ is a unit of quantum conductance [1]. Various quantized conductance behavior has been observed for metal nanoconstrictions fabricated using a scanning tunneling microscope (STM) and mechanically controllable break junctions (MCBJ) technique. While detailed studies have been done for the conductance properties of the atom-size nanoconstrictions, the single molecular junction has not been studied satisfactorily. Several pioneering attempts, however, provided important information on the nature of the single molecular junction $[3,4]$. Smit et al. could determine the conductance of a single hydrogen molecule bridging between $\mathrm{Pt}$ electrodes at ultra low temperature in ultra high vacuum (UHV) [3]. They observed a clear $0.9 G_{0}$ feature in the conductance histogram by dosing hydrogen gas to the Pt nanogap. The conductance fluctuation, point contact spectroscopy [5] shot noise measurements [6], and theoretical calculation result [7-9] show that the $0.9 G_{0}$ feature is the conductance of a single hydrogen molecule bridging between Pt electrodes.

Adsorbed hydrogen on $\mathrm{Pt}$ is an attractive system for the conductance measurement of single molecules. It should be noteworthy that the hydrogen/Pt system can be controlled by the electrochemical potential of substrates. Hydrogen evolution reaction is catalyzed most effectively by Pt-group metals electrode [10]. When the electrochemical potential of the Pt electrode is kept at the hydrogen evolution potential, the Pt electrode surface is covered by hydrogen molecules and dissociated atomic hydrogen. Dissociated atomic hydrogen, which is the intermediate of hydrogen evolution reaction, adsorbs on the on-top site of the Pt surface. At the under potential deposited hydrogen (UPD) potential, hydrogen atoms adsorb on the hollow site. The adsorbed hydrogen atoms and molecules would affect the conductance behavior of Pt nanoconstrictions. In the present 
study, we studied the quantized conductance behavior of mechanically fabricated $\mathrm{Pt}$ nanoconstrictions under electrochemical potential control in $\mathrm{H}_{2} \mathrm{SO}_{4}$ (acid), $\mathrm{Na}_{2} \mathrm{SO}_{4}$ (neutral) and $\mathrm{NaOH}$ (alkaline) solutions. The effect of the hydrogen on the quantized conductance behavior of $\mathrm{Pt}$ nanoconstrictions was discussed to consider the structure of the nanoconstriction.

\section{Experimental}

An electrochemical scanning tunneling microscope (EC-STM) was used to fabricate metal nanoconstrictions. A STM tip was made of a Pt wire (diameter $\sim 0.25 \mathrm{~mm},>99.9 \%$ ) coated with wax to reduce a Faradic current in an electrolyte solution. The Pt polycrystalline electrode was prepared by a flame annealing and quenching method. The experiments were performed in an electrochemical cell in a chamber filled with high purity $\mathrm{N}_{2}$ gas $(>99.999 \%)$ to reduce the effect of oxygen in air. The electrochemical potential of the substrate and tip was controlled using a potentiostat (Pico-Stat, Molecular Imaging Co.) with respect to a $\mathrm{Ag} / \mathrm{AgCl}$ reference electrode. $\mathrm{A}$ Pt wire was used as a counter electrode. The electrolyte solutions were $0.05 \mathrm{M} \mathrm{H}_{2} \mathrm{SO}_{4}, 0.1 \mathrm{M}$ $\mathrm{Na}_{2} \mathrm{SO}_{4}$, and $0.1 \mathrm{M} \mathrm{NaOH}$.

Atomic scale Pt nanoconstrictions were created in the following manner. The tip was pressed into the substrate and then pulled out from the substrate at a typical rate of $50 \mathrm{~nm} / \mathrm{s}$ in the electrochemical cell. During the contact breaking, the Pt nanoconstriction was formed between the tip and substrate. Conductance measurements were carried out at the breaking process under an applied bias voltage of $20 \mathrm{mV}$ between the tip and substrate. The conductance histograms were obtained from 1000 individual conductance traces.

\section{Results}

Figure 1(a) shows the cyclic voltammograms (CV) of a polycrystalline Pt electrode in the 0.05 $\mathrm{M} \mathrm{H}_{2} \mathrm{SO}_{4}$ solution. The double layer regime extended from $0.1 \mathrm{~V}$ to $0.6 \mathrm{~V}$. When the electrochemical potential of the Pt electrode was kept at potential more positive than $0.6 \mathrm{~V}$, the 
oxygen evolution reaction occurred via the formation of an oxide layer on the Pt electrode. A reduction current due to the under potential deposited hydrogen was observed at a potential regime from $-0.2 \mathrm{~V}$ to $0.1 \mathrm{~V}$. The hydrogen evolution reaction occurred at a potential more negative than $-0.2 \mathrm{~V}$. The potentials of boundary of the hydrogen evolution, UPD, double layer, and oxygen evolution regimes were defined as $\Phi_{1}, \Phi_{2}$, and $\Phi_{3}$ to be $\mathbf{- 0 . 2 , 0 . 1}$ and $\mathbf{0 . 6} \mathbf{V}$ respectively. Figure 1 (b) and (c) show the $\mathrm{CV}$ obtained in the $0.1 \mathrm{M} \mathrm{Na}_{2} \mathrm{SO}_{4}$ and $0.1 \mathrm{M} \mathrm{NaOH}$ solutions. $\Phi_{1}, \Phi_{2}$, and $\Phi_{3}$ were -0.8, -0.4, and $\mathbf{0 . 3} \mathrm{V}$ for $0.1 \mathrm{M} \mathrm{Na}_{2} \mathrm{SO}_{4}$, and were -0.8, -0.5, and -0.3 $\mathbf{V}$ for $0.1 \mathrm{M} \mathrm{NaOH}$, respectively. Conductance of $\mathrm{Pt}$ nanoconstrictions was measured considering these boundary potentials of the double layer, UPD, and the hydrogen evolution potentials.

Figures 2 and 3 (a-c) show conductance traces and histograms of Pt nanoconstrictions when the electrochemical potential of the Pt nanoconstrictions was kept at the double layer potential. The conductance changed without showing any clear plateaus, which leads to the featureless conductance histograms. The conductance behavior did not change at UPD potential (see Fig. 2, 3(d-f)). When the electrochemical potential of the Pt nanoconstrictions was kept at the hydrogen evolution potential, the conductance behavior drastically changed from those at the double layer and UPD potentials. The conductance of the Pt nanoconstrictions changed in a stepwise fashion as shown in the trace (see Fig. 2(g-i)). Furthermore, the $0.5 G_{0}$ and $1 G_{0}$ plateau were frequently observed at these steps. As a result, the conductance histogram shows clear features around 0.5 and $1 G_{0}$ in the $0.05 \mathrm{M} \mathrm{H}_{2} \mathrm{SO}_{4}$ solution. In the $0.1 \mathrm{M} \mathrm{Na}_{2} \mathrm{SO}_{4}$ and the $0.1 \mathrm{M} \mathrm{NaOH}$ solutions, there are clear features around $1 G_{0}$ with a shoulder around $0.5 G_{0}$.

\section{Discussion}

These experimental results clearly showed that the quantized conductance behavior of the Pt nanoconstrictions depended on the electrochemical potential and solution $\mathrm{pH}$. The origin of the quantized conductance behavior of the Pt nanoconstrictions is discussed in the following.

At the double layer potential, the conductance traces did not show any clear plateaus and, thus, 
the conductance histograms were featureless. This conductance behavior is discussed considering the effect of molecular adsorption on the conductance of metal nanoconstrictions. While the conductance histogram of clean Pt nanoconstrictions shows a clear feature around $1.5 \sim 2 G_{0}$ $[7,11,12]$, a broad feature around $1 G_{0}$ is observed in the histogram with a bias voltage of $200 \mathrm{mV}$ in $\mathrm{N}_{2}+5 \% \mathrm{H}_{2}$ at room temperature [12]. The decrease in the conductance is attributed to the adsorption of gas molecules $\left(\mathrm{O}_{2}, \mathrm{CO}, \mathrm{H}_{2}\right.$, etc $)$. A similar conductance change is observed for $\mathrm{Cu}$ and Au nanoconstrictions in solution $[13,14]$. Adsorbed molecules change the electronic structure and/or scatter the conduction electrons of the metal nanoconstrictions, leading to the decrease in the conductance. At the double layer potential, a Pt electrode surface is covered by sulfate anions and water molecules in the $\mathrm{H}_{2} \mathrm{SO}_{4}$ solution $[15,16]$. Since sulfate anions and water molecules would adsorb on various sites of the Pt nanoconstriction, the variation of the adsorption states may lead to the loss of a fixed conductance value, which gives the featureless conductance histogram. At the UPD potential, the polycrystalline Pt electrode surface is covered by the UPD hydrogen and sulfate anions [10]. The adsorption of the UPD hydrogen and sulfate anions would make the conductance histogram featureless.

At the hydrogen evolution potential, the conductance histogram showed clear features around 0.5 and $1 G_{0}$ in the $\mathrm{H}_{2} \mathrm{SO}_{4}$ solution, while the histograms showed a $1 G_{0}$ feature with a shoulder around $0.5 G_{0}$ in the $0.1 \mathrm{M} \mathrm{Na}_{2} \mathrm{SO}_{4}$ and $0.1 \mathrm{M} \mathrm{NaOH}$ solutions. At the hydrogen evolution potential, the Pt electrode surface is covered by the UPD hydrogen, the over potential deposition (OPD) atomic hydrogen, and evolved hydrogen molecules. There are no sulfate anions and water molecules on the Pt electrode surface $[10,16]$. Under the condition that only hydrogen species adsorb on the Pt nanoconstriction, a certain atomic configuration with the conductance of $1 G_{0}$ was stabilized. Similar $0.9 G_{0}$ feature was also observed for the Pt nanoconstrictions under hydrogen dosing in UHV. The feature is attributed to the hydrogen molecule bridging between two Pt electrodes $[3,5,6]$. Therefore, the observed $1 G_{0}$ feature would originate from a hydrogen molecule bridging two Pt electrodes in solutions. Figure 4 (a) shows proposed model of the structure showing 
conductance of $1 G_{0}$.

The $0.5 G_{0}$ feature observed in the present system has never been reported. It is noteworthy that the $0.5 G_{0}$ feature was clearly observed in the $0.05 \mathrm{M} \mathrm{H}_{2} \mathrm{SO}_{4}$ solution, while only a shoulder around 0.5 $G_{0}$ was observed in the $0.1 \mathrm{M} \mathrm{Na}_{2} \mathrm{SO}_{4}$ and $0.1 \mathrm{M} \mathrm{NaOH}$ solutions. The origin of the $0.5 G_{0}$ feature is discussed based on the difference of the $0.5 G_{0}$ feature among the solutions. The coverage of the OPD hydrogen on the Pt electrode is evaluated in acid, neutral and alkaline solutions by current-potential curves and infrared spectroscopy $[17,18]$. The coverage is more than 1 monolayer (ML) and below $0.1 \mathrm{ML}$ in the $\mathrm{H}_{2} \mathrm{SO}_{4}$ and $\mathrm{NaOH}$ solutions, respectively [17]. Therefore, the coverage of the OPD hydrogen would play a decisive role in appearance of the $0.5 G_{0}$ feature. The conductance of nanoconstrictions depends not only on the electronic structure of the point contact of atoms or molecules bridging the two electrodes, but also on that of the stem part of the contact. At higher coverage of the OPD hydrogen, the stem part of the Pt electrode would be also covered by the OPD hydrogen. The adsorbed OPD hydrogen is expected to change the electronic structure of the stem part of the Pt electrodes, leading to the change in the conductance of the metal or molecule bridging Pt electrodes. In addition to the possibility of the contribution of the OPD hydrogen, a hydrogen molecule bridging Pt electrodes could be also considered as the origin of the $0.5 G_{0}$ feature (see Fig. 4 (b)). One or two hydrogen molecules could bridge between Pt electrodes at higher coverage of the OPD hydrogen. Additional adsorption of hydrogen would decrease the conductance of the hydrogen molecule bridge with the conductance of $1 G_{0}$ (Fig. 4 (a)). Figure 4(c) shows another structure model showing the conductance of $0.5 G_{0}$, in which two hydrogen molecules bridge between Pt electrodes. At this moment, the origin of the $0.5 G_{0}$ and $1 G_{0}$ features is not clear, since we have not directly observed hydrogen atom or molecule at the nano constriction. Systematic investigation, such as electrochemical potential, solution $\mathrm{pH}$, metal dependence, would clarify the origin of the feature, and is in progress in our laboratory.

\section{Conclusion}


We studied the quantized conductance behavior of Pt nanoconstrictions under electrochemical potential control in $0.05 \mathrm{M} \mathrm{H}_{2} \mathrm{SO}_{4}, 0.1 \mathrm{M} \mathrm{Na}_{2} \mathrm{SO}_{4}$ and $0.1 \mathrm{M} \mathrm{NaOH}$ solutions. The conductance of Pt nanoconstrictions was measured at the double layer, the UPD, and the hydrogen evolution potential. Conductance histograms were featureless at the double layer or the UPD potential. At the hydrogen evolution potential, there were clear features in the histograms around 0.5 and $1 G_{0}$ in the $0.05 \mathrm{M} \mathrm{H}_{2} \mathrm{SO}_{4}$ solution, while $1 G_{0}$ with a shoulder around $0.5 G_{0}$ in $0.1 \mathrm{M} \mathrm{Na}_{2} \mathrm{SO}_{4}$ and $0.1 \mathrm{M}$ $\mathrm{NaOH}$ solutions. Contribution of a hydrogen molecule bridging Pt electrodes was considered as the origin of the $0.5 G_{0}$ and $1 G_{0}$ features in the histogram.

\section{Acknowledgements}

This work was partially supported by a Grant-in-Aid for Scientific Research A (No. 16205026) and Grant-in-Aid for Scientific Research on Priority Areas "Electron transport through a linked molecule in nano-scale" (No. 17069001) from MEXT. 


\section{References}

[1] N. Agrait, A. L. Yeyati and J. M. van Ruitenbeek, Physics Reports 377 (2003) 81.

[2] Jan van Ruitenbeek, Elke Scheer and Heiko Weber, in: "Introducing Molecular Electronics", G.

Cuniberti, G. Fagas, and K. Richter, eds., Springer Lecture Notes in Physics, 253 (2005).

[3] R. H. M. Smit, Y. Noat, C. Untiedt, N. D. Lang, M. C. van Hemert, and J. M. van Ruitenbeek, Nature 419 (2002) 906.

[4] B. Xu, and N. J. Tao, Science 301 (2003) 1221.

[5] D. Djukic, K. S. Thygesen, C. Untiedt, R. H. M. Smit, K. W. Jacobsen, and J. M. van Ruitenbeek, Phys. Rev. B 71 (2005) 161402.

[6] D. Djukic and J. M. van Ruitenbeek, Nano Lett. 6 (2006) 789.

[7] J. C. Cuevas, J. Heurich, F. Pauly, W. Wenzel, and G. Schön, Nanotechnology 14 (2003) R29.

[8] K. S. Thygesen and K.W. Jacobsen, Phys. Rev. Lett. 94 (2005) 036807.

[9] V. M. García-Suárez, A. R. Rocha, S. W. Bailey, C. J. Lambert, S. Sanvito, and J. Ferrer, Phys. Rev. B 72 (2005) 045437.

[10] G. Jerkiewicz, Prog. Surf. Sci. 57 (1998) 137.

[11] R. H. M. Smit, C. Untiedt, A. I. Yanson, and J. M. van Ruitenbeek, Phys. Rev. Lett. 87 (2001) 266102.

[12] K. Yuki, S. Kurokawa and A. Sakai, Jpn. J. Appl. Phys. 40 (2001) 803.

[13] A. Bogozi, O. Lam, H. He, C. Li, N. J. Tao, Larry A. Nagahara, I. Amlani, and R. Tsui, J. Am. Chem. Soc. 123 (2001) 4585.

[14] H.X. He, C. Shu, C.Z. Li, and N.J. Tao, J. Electroanal. Chem. 522 (2002) 26.

[15] A.M. Funtikov, U. Stimming, and R. Vogel, J. Electroanal. Chem. 428 (1997) 147.

[16] K. Shimazu, and H. Kita, J. Electroanal. Chem. 341 (1992) 361.

[17] B. E. Conway, and L. Bai, J. Electroanal. Chem. 198 (1986) 149.

[18] N. Nanbu, F. Kitamura, T. Ohsaka, and K. Tokuda, J. Electroanal. Chem. 485 (2000) 128. 


\section{Figure captions}

Figure 1 : Cyclic voltammogram of a polycrystalline Pt electrode in (a) $0.05 \mathrm{M} \mathrm{H}_{2} \mathrm{SO}_{4}$, (b) $0.1 \mathrm{M}$ $\mathrm{Na}_{2} \mathrm{SO}_{4}$, (c) $0.1 \mathrm{M} \mathrm{NaOH} . \Phi_{1}, \Phi_{2}, \Phi_{3}$ are the potential of boundary between the hydrogen evolution and UPD, double layer, oxygen evolution.

Figure 2 : Typical conductance traces of Pt nanoconstrictions at the double layer (DL: a-c), the UPD (UPD: d-f) and the hydrogen evolution potential (OPD:g-i). These traces were measured in $0.05 \mathrm{M} \mathrm{H}_{2} \mathrm{SO}_{4}(\mathrm{a}, \mathrm{d}, \mathrm{g}), 0.1 \mathrm{M} \mathrm{Na}_{2} \mathrm{SO}_{4}(\mathrm{~b}, \mathrm{e}, \mathrm{h})$, and $0.1 \mathrm{M} \mathrm{NaOH}(\mathrm{c}, \mathrm{f}, \mathrm{h})$.

Figure 3 : Conductance histograms of Pt nanoconstrictions at the double layer (DL: a-c), the UPD (UPD: d-f) and the hydrogen evolution potential (OPD:g-i). These histograms were obtained in 0.05 $\mathrm{M} \mathrm{H}_{2} \mathrm{SO}_{4}(\mathrm{a}, \mathrm{d}, \mathrm{g}), 0.1 \mathrm{M} \mathrm{Na}_{2} \mathrm{SO}_{4}(\mathrm{~b}, \mathrm{e}, \mathrm{h})$, and $0.1 \mathrm{M} \mathrm{NaOH}(\mathrm{c}, \mathrm{f}, \mathrm{h})$.

Figure 4: Schematic models of the structure showing conductance of $1 G_{0}(\mathrm{a})$ and $0.5 G_{0}(\mathrm{~b}, \mathrm{c})$. (a) a hydrogen molecule bridging between Pt electrodes, (b) a hydrogen molecule bridging between $\mathrm{Pt}$ electrodes covered by the hydrogen. (c) two hydrogen molecules bridging between Pt electrodes. 
Figure1

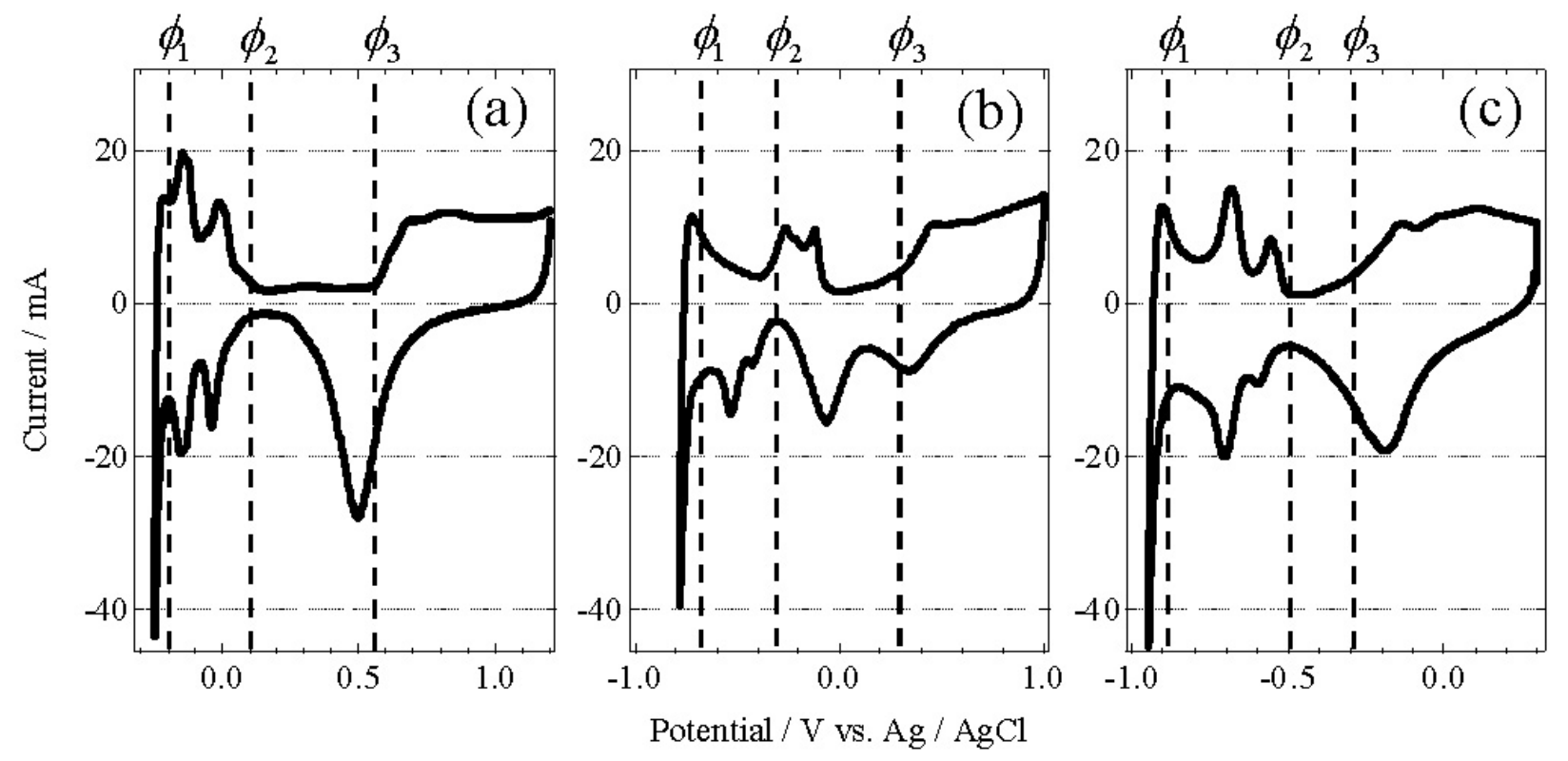


Figure2

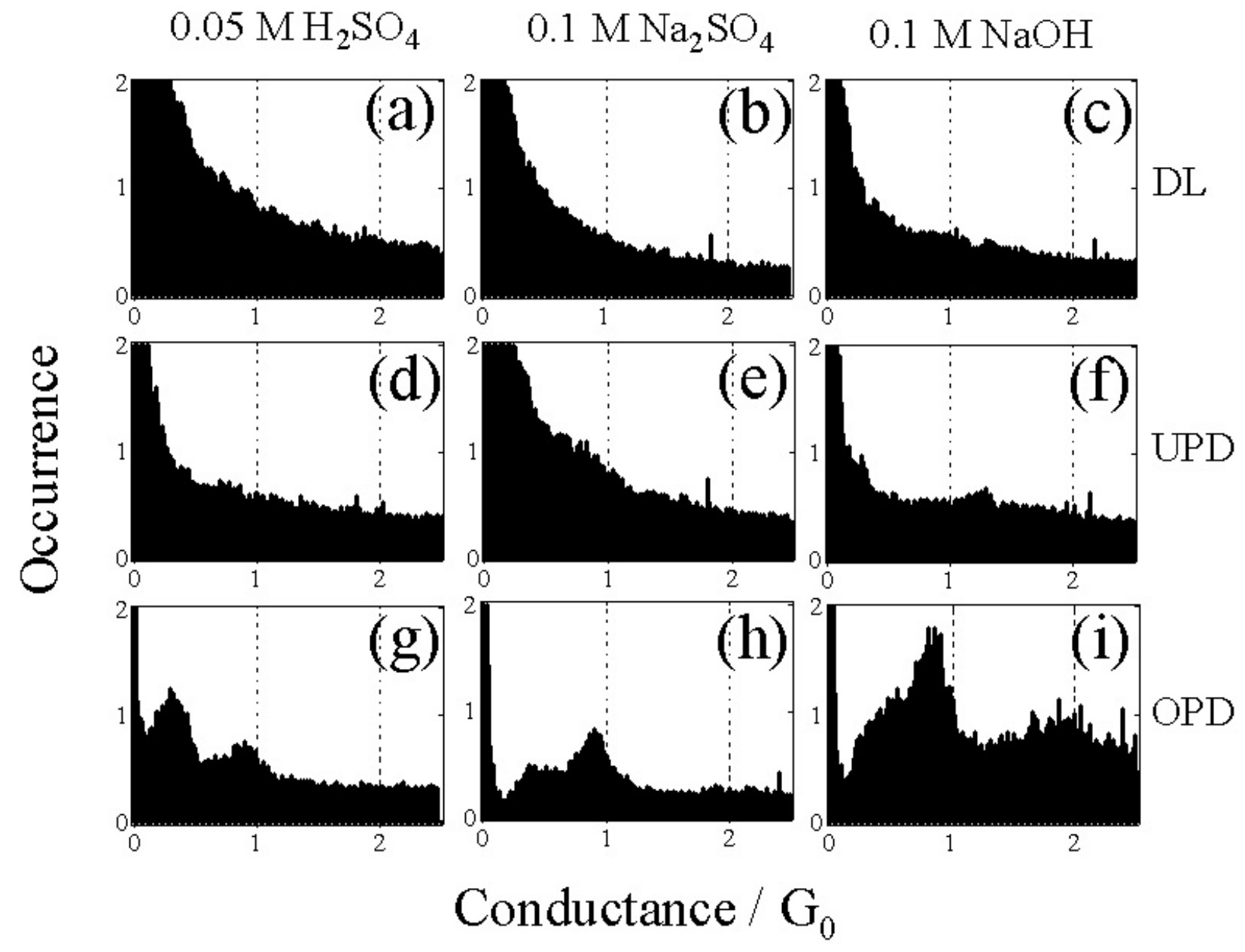


Figure3

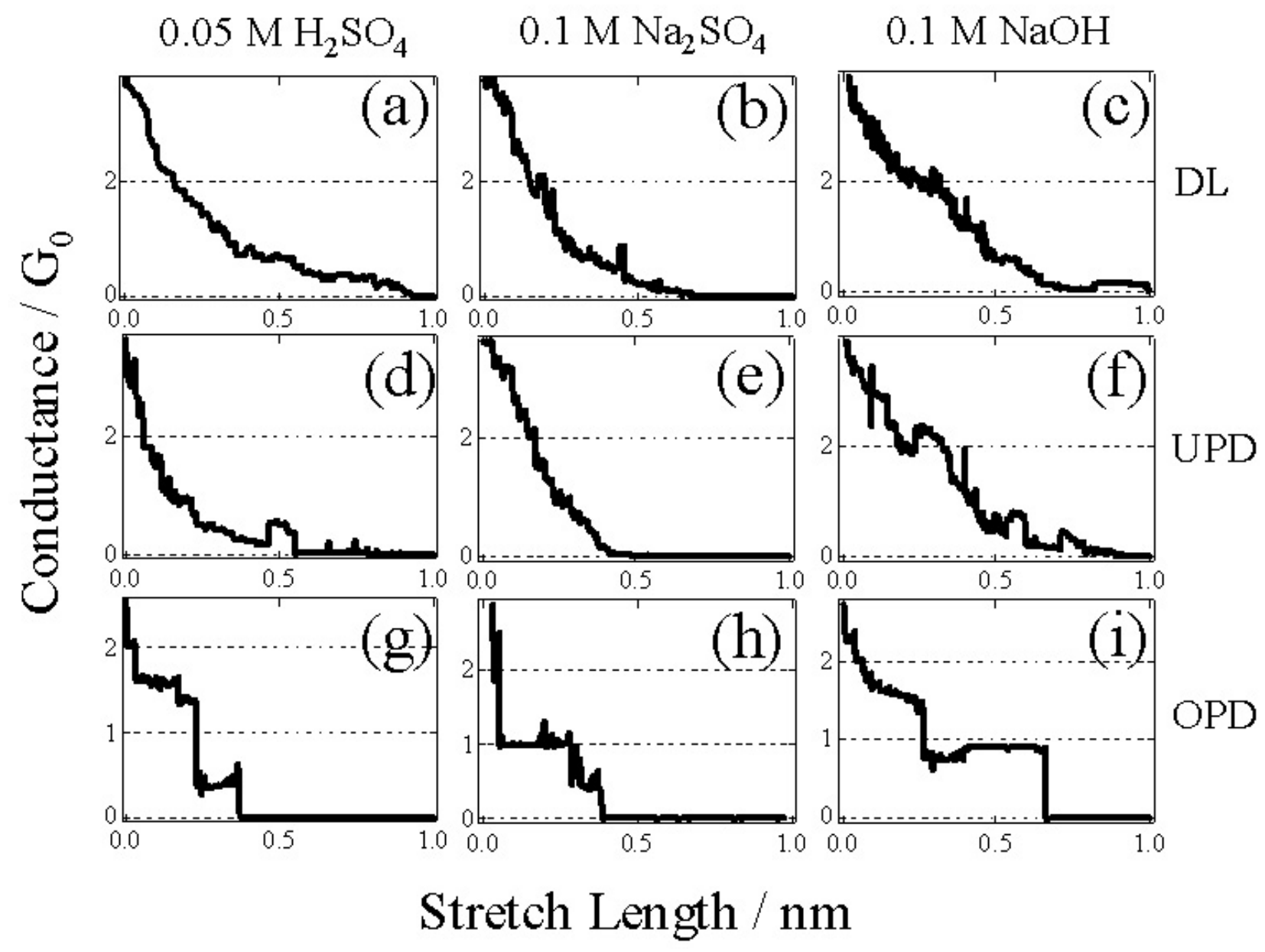


Figure4
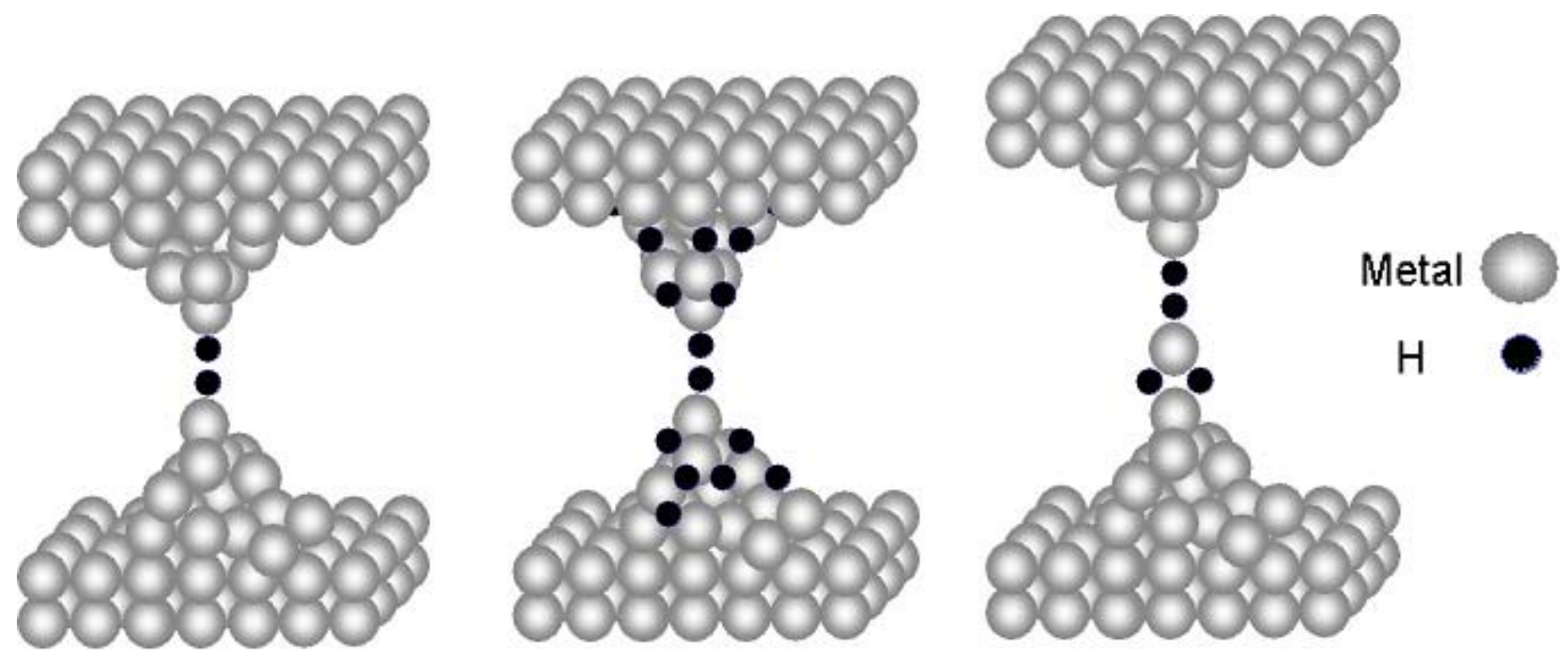